\title{
Migränepatienten: „Infarct-like-lesions“ sind keine Seltenheit
}

\section{Ob eine Migräne zu strukturellen Schäden und zwar in Form einer Ischämie im Gehirn führen kann, wird seit vielen Jahren diskutiert. Neuere MRI-Unter- suchungen zeigen, dass "infarct-like-lesions" gar nicht so selten sind. Doch welche klinische Bedeutung haben diese für den Migränepatienten?}

$D$ ie Abgrenzung zwischen Migräne und ischämischem Insult ist nicht immer einfach. „Auch gibt es Krankheitsbilder, bei denen man sowohl die typischen Charakteristika einer Migräne als auch die eines ischämischen Insults findet", sagte Professor Nils Peters, Neurologische Klinik, Universitätsspital Basel. Dazu gehört das CADASIL(Cerebral Autosomal-Syndrom Dominant Arteriopathy with Subcortical Infarcts and Leukencephalopathie)-Syndrom, eine genetische Erkrankung. Bei diesem Krankheitsbild finden sich ischämische Insulte beziehungsweise TIA (87\%) und eine Migräne mit Aura (38\%), außerdem nicht selten eine Demenz (59\%) und selten Krampfanfälle (10\%).

\section{Zeichen einer Mikroangiopathie}

Aber auch bei der nicht genetisch determinierten Migräne können nicht selten
Zeichen einer Mikroangiopathie auftreten. So fanden sich in einem Kollektiv von 4.500 Patienten im MRI hyperintensive "white matter lesions" bei $39 \%$ der Männer und 25\% der Frauen, wobei das Risiko bei einer Migräne um den Faktor 1,4 bei Männern und um den Faktor 1,9 bei Frauen erhöht war. Frauen sind also häufiger betroffen. Diese Veränderungen sind in der Regel infratentoriell, bei Frauen vorwiegend im Kleinhirn lokalisiert. Diese „Infarct-like-lesions" treten unabhängig von den klassischen kardiovaskulären Risikofaktoren auf. Bei Verlaufsbeurteilungen zeigte sich häufig auch eine Progression, das heißt, es traten im weiteren Verlauf neue Läsionen auf. Dabei bestand jedoch keine Assoziation mit der Kopfschmerzfrequenz oder der Blutdruckhöhe.

Doch wie entstehen diese Läsionen? Diskutiert werden verschiedene patho- genetische Mechanismen: Hypoperfusion, Vasoreaktivität, Endothelschädigung, Thrombosierungen beziehungsweise Embolien und Störungen der Bluthirnschranke.

\section{Welche klinische Relevanz?}

Die entscheidende Frage aber ist: Welche klinischen Auswirkungen haben diese „Infarct-like-lesions“ für Migränepatienten? „Ein klinisches Ereignis mit neurologischen Ausfällen findet sich in der Regel nicht", so Peters. Auch gebe es bisher keinerlei Hinweise dafür, dass diese Veränderungen mit kognitiven Störungen einhergehen oder zu solchen führen können. Doch stelle sich angesichts dieser Befunde die Frage, ob man bei der Migräne weiterhin von einer episodischen Erkrankung ausgehen dürfe oder ob es sich nicht doch um eine chronische Erkrankung handele, was auch Implikationen für die Therapie habe und zwar dahingehend, dass der Verhinderung von Migräneattacken ein vorrangiger Stellenwert zukommen müsse.

Dr. med. Peter Stiefelhagen

"Spotlight Migräne und vaskuläre Erkrankungen", 88. Kongress der Deutschen Gesellschaft für Neurologie, Düsseldorf, 23. - 26.9.2015

\section{Lebensbedingungen in der Kindheit beeinflussen das spätere Schlaganfallrisiko}

\author{
Kinder, die in ungünstigen sozioökonomischen Verhältnissen aufwachsen, \\ haben ein erhöhtes Risiko, später einen Schlaganfall zu erleiden. Ein Einfluss- \\ faktor dabei ist die Zahl älterer Geschwister, wie eine Fall-Kontroll-Studie in \\ Ludwigshafen zeigt.
}

D ie Schlaganfallinzidenz und -mortalität ist in Ländern mit niedrigem oder mittlerem Einkommensniveau höher als in Ländern mit hohem Einkommensniveau. Herkömmliche kardiovaskuläre Risikofaktoren erklären die Assoziation zwischen sozioökonomischen Verhältnissen sowie Inzidenz und Mortalität des Schlaganfalls nur teilweise. Deshalb untersuchte die Fall-KontrollStudie GENESIS unter anderem die Assoziation von sozioökomischen Parame- tern in Kindes-, Jugend und Erwachsenenalter bei 470 Patienten nach einem ischämischen Schlaganfall und 807 Kontrollen aus Ludwigshafen.

Wie Professor Armin Grau, Chefarzt der Neurologie am Klinikum Ludwigshafen, berichtete, bestätigte die Auswertung zunächst die bekannten Schlaganfallrisikofaktoren wie Hypertonie, Diabetes, Hypercholesterinämie, Vorhofflimmern, pAVK, Rauchen, hoher Alkoholkonsum und fehlende körperliche
Aktivität, die alle in der Gruppe der Schlaganfallpatienten häufiger waren als in der Kontrollgruppe.

Korrigiert um diese Einflussfaktoren ergab sich in der Adoleszenz und im späteren Erwachsenenalter eine Erhöhung des Schlaganfallrisikos insbesondere bei einer fehlenden Berufsausbildung $(\mathrm{OR}=$ 1,93 als Faktor in der Adoleszenz bzw. $\mathrm{OR}=1,99$ als Faktor im Erwachsenenalter). Im Erwachsenenalter waren außerdem eine Arbeitslosigkeit über sechs Monate und der Familienstand (ledig, geschieden oder verwitwet) mit einem erhöhten Schlaganfallrisiko assoziiert (OR $=1,52$ bzw. 1,63).

In der multivariaten Analyse für die Kindheit konnten die Autoren als weitere - von den bekannten Risikofaktoren unabhängige - Risikofaktoren identifizieren: mehr als drei Geschwister $(\mathrm{OR}=$ 1,48), das Fehlen einer Toilette in der 